\title{
ARTICLE
}

\section{The Effects of Selenium Nanoparticles (SeNPs) on Ruminant}

\author{
Khandsuren Badgar 1, 2 * and József Prokisch ${ }^{1,2}$ \\ ${ }^{1}$ Institute of Animal Science, Biotechnology and Nature Conservation, Faculty of Agricultural and \\ Food Sciences and Environmental Management, University of Debrecen, Hungary \\ ${ }^{2}$ Doctoral School of Animal Science, University of Debrecen, Hungary
}

\begin{abstract}
This study examines the role of selenium and the effects of selenium nanoparticle-enriched food supplementation especially in ruminant animals. Selenium is an essential element that has multiple biological activities. Particularly it is important for many physiological processes, especially for the functions of reproductive and immune systems, metabolism of thyroid hormones, as well as antioxidant defense. Also, the presence of selenium nanoparticles in the diet provide a high content of selenium in milk and meat due to its high bioavailability and low toxicity, which is essential for humans.
\end{abstract}

Keywords: Selenium nanoparticles; SeNPs; ruminant; functional properties;

\section{INTRODUCTION}

Selenium was discovered in 1817 by the Swedish chemist Jöns Jacob Berzelius. In the beginning, the scientific community thought that selenium was toxic, but in the 1950s it was found that is a biologically essential element lends much support to health maintenance and disease prevention of humans and other all living organisms that is a major structural component of some enzymes and hormones. Actually, its nutritional value depends mainly on the dose and type of selenium species present in the source, such as plant, soil, food, and food supplements. The common dietary supplements of selenium are inorganic selenium (selenate, selenite, selenide, elemental selenium), organic selenium (selenocystine, Semethylselenocysteine), and elemental selenium. A type of nanoparticle (SeNPs) has higher bioavailability [1-3], much lower toxicity [2], and a stronger absorption ability [4] than other general forms, and its applicability is dominated by several significant characteristics such as size, shape, atomic arrangement, structure, and surface charge. On the other hands, its biological properties and application depend on the ability to synthesize particles with different shape, monodispersity, chemical composition, and size.

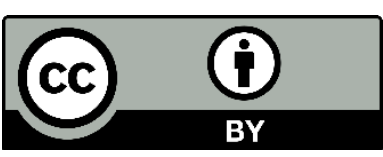

The Author(s). 2020 Open access This article is distributed under the terms of the Creative Commons Attribution 4.0 International License (https://creativecommons.org/licenses/by/4.0/), which permits unrestricted use, distribution, and reproduction in any medium, provided you give appropriate credit to the original author(s) and the source, provide a link to the Creative Commons license, and indicate if changes were made. 
Therefore, selenium nanoparticle has recently attracted even more attention in many scientific circles, and it has been widely used in food supplements and nano-medicine. Because, selenium nanoparticles have functional properties for treatment and prevention, which have important roles in the feed digestibility [5-7], reproductive system [812], antioxidant system [1,3], immune systems [13], anticancer [14-20], antibacterial [21-24] and intoxication process [25]. The biomedical applications and food supplements of selenium nanoparticles have been increasing based on their properties of non-toxic, high positive effects for prevention and treatment. In this review, we aimed to integrate the functional properties of nano-size selenium and applications of its food supplement for ruminant animals.

\section{THE EFFECT OF SELENIUM NANOPARTICLES ON RUMEN FERMENTATION AND DIGESTION}

Rumen fermentation is a fundamental and special process of the ruminant metabolism and it determines the assimilation and intake of nutrients from ingested food. Some studies reported the effect of selenium nanoparticles on rumen microbial fermentation. Wenjuan Xun et al., reported that rumen fermentation and feed utilization improved by nano-size selenium supplementation in the basal diet. Selenium nanoparticles could also stimulate rumen microbial activity, digestive microorganisms, or enzyme activity [5]. These authors studied the effect of nano-size selenium and selenium yeast supplements on food digestibility, rumen fermentation, and found that urine purine derivatives in male sheep demonstrated a decrease in rumen $\mathrm{pH}$, concentration of ammonia nitrogen, level of propionate, and acetate: propionate ratio. The results showed that ruminal fermentation and feed conversion efficiency improved in the nano-size selenium group, and total volatile fatty acid (VFAs) of rumen increased in the supplemented groups like nano-size selenium and yeast-selenium [26].

In another study, selenium supplement improved microbial activity, contributed to higher bioavailability, catalytic efficiency, and strong adsorbing ability. Selenium in ruminant diets is not solely for the animal per selenium but also for rumen bacteria capable of metabolizing part of it $[27,28]$. Most of the selenium in the rumen fluids of sheep under supplementation is insoluble, indicating the influence of the rumen environment on selenium bioavailability [27,29].
Also, ruminal microorganisms, such as Lactic acid bacteria, Bifidobacteria, and Protozoa (ciliates) alter the bioavailability of received selenium [7]. For example; the study of sheep experiment showed the opposite effect, with higher acetic and isovaleric acids [30] and the total concentration of VFAs, and increased protozoa population, with a greater proportion of Diplodinium [31]. Kheradmand et al. investigated the effect of nano-size selenium on the antifungal activity of probiotic bacteria. After exposure to nano-size selenium-enriched Lactobacillus Plantarum and Lactobacillus Johnsonii, a greater decline in the viability of Candida albicans than after its exposure to nonSe-enriched Lactic acid bacteria was observed. The antifungal activity of both bacterial strains increased through selenium [32].

Faixová Z. et al., reported that selenium affects the activities of Gamma-glutamyl transferase (GGT) and glutamine dehydrogenase $(\mathrm{GDH})$ in the rumen fluid of the sheep. These enzymes were significantly higher after oral administration of selenized yeast, alkaline phosphatase, and aspartate aminotransferase than when sodium selenite $\left(\mathrm{Na}_{2} \mathrm{SeO}_{3}\right)$ was used [33].

The absorption of selenium in the gastrointestinal tract is significantly different between ruminants and non-ruminants. This process is much lower in ruminants than in nonruminants. For example, orally administered selenium was only 34 percent in sheep compared with 85 percent in swine [34]. Low absorption of ruminants is believed to be the result of reduction of dietary selenium to insoluble forms, such as elemental selenium or 
selenides in the rumen environment [35]. The microbial digestion in the rumen and reticulum precedes digestion in the abomasum and small intestine [36].

\section{THE EFFECT OF NANO-SIZED ELEMENTAL SELENIUM ON REPRODUCTION AND REPRODUCTIVITY}

Selenium plays an important role in the synthesis of testosterone and sperm, and sperm motility and its deficiency affects reproductive parameters, such as abnormal mitochondria in spermatozoa and growth performance in male animals. Further oxidative stress affects the fertility potential of spermatozoa by lipid peroxidation, which can lead to sperm dysfunction [11]. Selenium nanoparticle supplementation increases selenium concentration in testes, and testicular and glutathione peroxidase (GPx) activity, which has a protective effect on membrane integrity of the mitochondria. Shi L. et al. reported the effects of nano-size selenium in the diet $(0.3$ $\mathrm{mg} / \mathrm{kg}$ body weight for 12 weeks) on the testes ultrastructure, ejaculate quality, and glutathione peroxidase activity in goat. The results of this study showed that the level of selenium in testes and the activity of GPx and ATPase in the ejaculate significantly increased in the nanosize selenium supplemented compared to the unsupplemented group. And the quality of ejaculates, such as volume, density, motility, and $\mathrm{pH}$ was not affected, but the percentage of abnormal spermatozoa and damage of spermatozoon membrane was significantly higher in the control group [10].

For female animals, selenium affects pregnancy rates, fertility, embryonic implantation, placenta retention, ovarian cysts, the incidence of metritis, and abortions and stillborn. The increase of selenium concentration prepartum in blood correlates with anoestrus/silent oestrus decreasing postpartum [12].

The selenium deficiency in grazing beef cattle was the sole cause of abortion in cows
[37]. Selenium and vitamin E administration reduces the incidence of retained placenta [9] and it can decrease incidence of metritis and ovarian cysts [10].

In addition, selenium nanoparticle contributes to increasing hair follicle development (wool production) and fetal growth. Generally, selenium intake stimulates the proliferation, migration, and morphogenesis of hair follicle cells through insulin-like growth factor receptor (IGF-1R) during ontogenetic development [38]. Insulin-like growth factor (IGF-1) represents peptide hormone produced in the liver, which is formed as a consequence of growth hormone $(\mathrm{GH})$ released from the pituitary gland, which subsequently stimulates IGF-1 production in liver. IGF-1 is, therefore, a mediator for some of the GH functions, and is thus involved in growth and anabolism. IGF-1 mediates its effects by binding at the specific receptor [39]. The study of Xiaoying $\mathrm{Wu}$ et al. found that nano-size selenium supplemented cashmere goats (basal diet with $0.5 \mathrm{mg} / \mathrm{kg}$ ) improved the development of secondary follicles and promoted the growth of the fetus. In the nano-size selenium supplemented group, GSH-Px, IGF-1, and IGF-1R, as well as the activities of GSH-Px and SOD, were significantly higher, but the MDA level was significantly lower. This was attributed to the influencing antioxidant status (T-AOC) in the fetal skin. The weights of fetal liver, kidney tissues, fetus, and placenta were thus significantly higher in nano-size selenium supplemented groups than in the control group [40].

\section{THE EFFECT OF NANO-SIZED ELEMENTAL SELENIUM ON RUMINANT HEALTH}

Selenium plays in many physiological functions, which include its ability to protect against certain heart diseases, muscle functioning and development, boost the strength of the immune system (GPx1, TR1), antioxidant activity (GPx), antimicrobial activity, anti-carcinogenic effect, maximize thyroid function (DIO1, DIO2, and DIO3), and 
intoxication process. Selenium is generally accumulated in immune response organs, such as the spleen, liver, and lymph nodes, which has shown to stimulate the formation of antibodies and the activity of the $\mathrm{T}$ helper cells along with cytotoxic $T$ and Natural Killer cells [41]. Selenium supplementation in diet can improve the expression of various humoral and cellular immune responses [42]. The colostrum plays a fundamental role in the passive transfer of immunity from the colostrum to the newborns.

Rasool Kachuee et al. published findings of the effect of organic, inorganic, and nanosize selenium supplements at the final stage of pregnancy on selenium (Se), zinc $(\mathrm{Zn})$, copper $(\mathrm{Cu})$, and iron $(\mathrm{Fe})$ concentrations of Khalkhali goats and placental, colostrum, and milk transfer of these trace minerals from goats to their kids. They used forty pregnant goats, which weree divided into an unsupplemented group, selenomethionine, nano-size selenium, and sodium selenite supplemented groups. The results showed that the whole blood and serum total selenium concentration was higher in the nano-size selenium supplemented group than other groups, which but decreased in their kids. Also copper concentration was greater, zink concentration was totally vice versa in the whole blood, serum, and colostrum, and iron concentration increased at birth and the first week of kids of selenium supplemented goats [43].

Also, Gholam Ali Kojouri et al. studied the effect of some selenium types, such as sodium selenite and nano-size selenium on iron (Fe) homeostasis and the expression of transferrin and its receptor-binding protein genes on sheep for 20 days. The results of this study showed that serum iron concentration decreased in the beginning to the middle stages of the experiment, TIBC level increased and the expression of transferrin and its receptor genes significantly increased during the experimental period in both groups [44]. In addition, the authors reported selenium nanoparticles as having a stronger stimulatory effect on intracellular activities in another article. Particularly, they have compared the effect of nano-size selenium and sodium selenite on characteristics of neutrophils in sheep for 30 days. However, nano-size selenium supplemented sheep dramatically increased the chemotactic and respiratory burst activities than the sodium selenite supplemented group [13].

The antioxidant properties of selenium related to its several genes, which include SEPP1, SEP15, SELENBP1, CAT, TRXNRD1-2, PRDX1-6, GPX, and SOD [45]. The glutathione peroxidase neutralizes hydrogen peroxide and organic hydroperoxides in the intracellular and extracellular compartments. Especially, the main activity of 1,2 , and 3 types is to protect against oxidative damages, antioxidants in plasma, and to reduce lipid hydro peroxidase. Shi et al. studied the antioxidant effect of inorganic, organic, and elemental nano-size selenium in growing weaned TAIHANG strain black male goats for 90 days. They determined the body weight (BW), average daily gain (ADG), serum glutathione peroxidase (GSH-Px), superoxide dismutase (SOD), catalase (CAT), and malondialdehyde (MDA) activity, and selenium concentration. The final BW increased $(\mathrm{P}<0.05)$, and selenium concentration of whole blood, serum and tissue, and serum antioxidant activity were affected in all selenium supplemented group as compared to the unsupplemented group. Also ADG was greater $(\mathrm{P}<0.05)$ in nano-size selenium and Seyeast supplemented groups. In addition, serum GSH-Px, SOD, and CAT were higher $(\mathrm{P}<0.05)$ in nano-size selenium supplemented group than in the supplemented groups with sodium selenite and Se-yeast. Se level of whole blood, serum, and some organs were higher in only nano-size selenium supplemented group [3].

Another study by Sirous Sadeghian et al. reported the effects of nano-size selenium (orally $1 \mathrm{mg} \mathrm{kg}^{-1}$ body weight) and sodium selenite (orally $1 \mathrm{mg} \mathrm{kg}^{-1}$-Se body weight) on the activity of neutrophils and hematological parameters in sheep for 30 days. The level of thiobarbituric acid reactive substances (TBARS) in plasma was significantly higher $(\mathrm{P}<0.05)$ in both selenium added groups than the control group. While white blood cell (WBC) count significantly increased in the nano-size selenium supplemented group, but the packed cell volume (PCV) and red blood cell (RBC) count were not significantly different in all groups. But in the nano-size 
selenium supplemented group, WBC was significantly higher on days 20 and 30 , and for sodium selenite supplemented group WBC increased significantly just in 20 days. The neutrophil counts had significantly increased and the lymphocyte counts significantly decreased on 10 days in only nano-size selenium supplemented groups, and on days 20 and 30 in both selenium supplemented groups compared to the control group [46].

Selenium poisoning occurs if the selenium level is very high in the feed, soil, pasture, and water, and if the selenium level is very low is in them, selenium deficiency occurs in both humans and animals. It mostly occurs in ruminants especially kids, lambs, and calves, which is called white muscle disease (WMD) or nutritional myopathy in Veterinary Science. Many scientists have reported about it, namely, Muller et al. recorded that a low and deficient dietary selenium concentration are prone to many problems, including white muscle disease in lambs and calves, calf pneumonia, infertility, and exudative diathesis in chickens and other animals [47].

Actually, selenium bioavailability varies depending on several factors such as chemical

\section{CONCLUSIONS}

Selenium nanoparticles are a good additive material for the functionalization of feed supplements. The systematic consumption of selenium nanoparticles and enriched feed supplements can increase the productivity of ruminants, also they can protect them from some disorders. It is reported that selenium absorption is much lower in ruminants than in

\section{REFERENCES}

1. Wang $\mathrm{H}$, Zhang J, Yu H., Elemental selenium at nano size possesses lower toxicity without compromising the fundamental effect on selenoenzymes: comparison with selenomethionine in mice. Free Radical Biology and Medicine. 2007, 42(10):1524-1533.

2. Zhang $\mathrm{J}$, Wang $\mathrm{X}, \mathrm{Xu} \mathrm{T}$., Elemental selenium at nano size (Nano-Se) as a forms, solubility, other dietary components, physiological status of the body [48]. Therefore, selenium concentration in whole blood, daily uptake (dosage), and chemical type are very important. Food supplement containing a suitable dose and the suitable type of selenium, especially elemental type, affects many of the above physiological functions and prevents many risks. Some studies have indicated to the blood selenium amount and daily intake value. For example; The Diagnostic Center for Population and Animal Health of Michigan State University determined the whole blood selenium concentration of ruminants, including sheep (lambs-adults) 120-350 ng/ml, goats (kidsadults) $170-300 \mathrm{ng} / \mathrm{ml}$, and cattle (calves till 30 days-adults) $100-300 \mathrm{ng} / \mathrm{ml}$. And for daily intake of selenium, National Research Council recommended $100 \mu \mathrm{g} \cdot \mathrm{kg}^{-1}$ dry matter for beef cattle and calves, $300 \mu \mathrm{g} \cdot \mathrm{kg}^{-1}$ dry matter [49] for dairy cattle, and $0.1-0.2 \mathrm{mg} \cdot \mathrm{kg}^{-1}$ dry matter [50] for sheep. For goats, the recommended daily intake is $0.1 \mathrm{mg} \cdot \mathrm{kg}^{-1}$ dry matter of feed [51].

monogastric animals. However, the study of nano-sized selenium absorption has not been completed. Therefore, the studies of feed supplement with selenium nanoparticles and their application, bioavailability, absorption, and accumulation for ruminants are needed to be continued in the future.

potential chemopreventive agent with reduced risk of selenium toxicity: comparison with Semethylselenocysteine in mice. Toxicol Science. 2008, 101(1): 22-31.

3. Shi L, Xun W, Yue W, Zhang C, Ren Y, Shi L,. Effect of sodium selenite, Se-yeast and nano-elemental selenium on growth performance, Se concentration and 
antioxidant status in growing male goats. Small Ruminant Research. 2011, 96(1): 49-52.

4. Wang RR, Pan XJ, Peng Z., Effects of heat exposure on muscle oxidation and protein functionalities of pectoralis majors in broilers. Poultry Science, 2009, 88:1078-1084.

5. Wenjuan Xun, Liguang Shi, Wenbin Yue, Chunxiang Zhang, Youshe Ren, Qiang Liu, Qian Wang, Lei Shi., Effect of elemental nano-selenium on feed digestively, rumen fermentation, and purine derivatives in sheep. Animal Feed Science and Technology, 2010, 163(24):136-42.

6. Wenjuan Xun, Liguang Shi, Wenbin Yue, Chunxiang Zhang, Youshe Ren, Qiang Liu., Effect of high-dose nano-selenium and selenium-yeast on feed digestibility, rumen fermentation, and purine derivatives in sheep. Biological Trace Element Research. 2012,150(1-3):130136.

7. Galbraith ML, Vorachek WR, Estill CT, Whanger PD, Bobe G, Davis TZ, Hall JA., Rumen microorganisms decrease bioavailability of inorganic selenium supplements. Biological Trace Element Research, 2016, 171:338-343.

8. Wilde D., Influence of macro and micro minerals in the peri-parturient period on fertility in dairy cattle. Animal Reproduction Science, 2006, 96:240249.

9. Spears JW, Weiss WP., Role of antioxidants and trace elements in health and immunity of transition dairy cows. Journal of Veterinary Science, 2008, 176:70-76.

10. Shi Li-guang, Yang Ru-jie, Yue Wen-bin, Xun Wen-juan, Zhang Chun-xiang, Ren You-she, Shi Lei, Lei Fu-lin., Effect of elemental nano-selenium on semen quality, glutathione peroxidase activity, and testis ultrastructure in male Boer goats. Animal Reproduction Science, 2010, 118(2): 248-254.

11. Badade ZG, More K, Narshetty J., Oxidative stress adversely affects spermatogenesis in male infertility.
Biomedical Research, 2011, 22(3):323328.

12. Fernandes AP, Wallenberg M, Gandin V, Misra S, Tisato F, Marzano C, Rigobello MP, Kumar S, Björnstedt M., Methylselenol formed by spontaneous methylation of selenide is a superior selenium substrate to the thioredoxin and glutaredoxin systems. PLoS ONE, 2012, 7(11): e50727.

13. Gholam Ali Kojouri, Sirous Sadeghian, Abdonnaser Mohebbi, Mohammad Reza Mokhber Dezfouli., The effects of oral consumption of selenium nanoparticles on chemotactic and respiratory burst activities of neutrophils in comparison with sodium selenite in sheep. Biological Trace Element Research, 2012, 146(2):160-166.

14. Chen T, Wong YS, Zheng W, Bai Y, Huang L., Selenium nanoparticles fabricated in Undaria pinnatifida polysaccharide solutions induce mitochondria-mediated apoptosis in A375 human melanoma cells. Colloids Surf B Biointerfaces, 2008, 67(1):26-31

15. Luo H, Wang F, Bai Y, Chen T, Zheng W., Selenium nanoparticles inhibit the growth of HeLa and MDA-MB-231 cells through induction of $\mathrm{S}$ phase arrest. Colloids Surf B Biointerfaces. 2012, 94:304-308

16. Ramamurthy $\mathrm{CH}$, Sampath KS, Arunkumar P, Kumar MS, Sujatha V, Prekumar K, Thirunavukkarasu C., Green synthesis and characterization of selenium nanoparticles and its augmented cytotoxicity with doxorubicin on cancer cells. Bioprocess and Biosystems Engineering, 2013, 36(8): 1131-1139

17. Pi J, Yang F, Jin H, Huang X, Liu R, Yang P, Cai J., Selenium nanoparticles induced membrane bio-mechanical property changes in MCF-7 cells by disturbing membrane molecules and Factin. Bioorganic \& Medicinal Chemistry Letters, 2013, 23(23):6296-6303

18. Zhang Y, Li X, Huang Z, Zheng W, Fan C, Chen T., Enhancement of cell permeabilization apoptosis-inducing activity of selenium nanoparticles by 
ATP surface decoration. Nano-medicine, 2013, 9(1):74-84

19. Bao P, Chen Z, Tai RZ, Shen HM, Martin FL, Zhu YG., Selenite-induced toxicity in cancer cells is mediated by metabolic generation of endogenous selenium nanoparticles. Journal of Proteome Research,2015, 14(2):1127-1136.

20. Liao W, Zhang R, Dong C, Yu Z, Ren J., Novel walnut peptide-selenium hybrids with enhanced anticancer synergism: facile synthesis and mechanistic investigation of anticancer activity. International Journal of Nanomedicine. 2016, 11:1305-1321

21. Srivastava N, Mukhopadhyay M., Green synthesis and structural characterization of selenium nanoparticles and assessment of their antimicrobial property. Bioprocess and Biosystems Engineering, 2015, 38(9):1723-1730

22. Sonkusre P, Singh Cameotra S. Biogenic selenium nanoparticles inhibit Staphylococcus aureus adherence on different surfaces. Colloids Surf B Biointerfaces. 2015, 1; 136:1051-1057

23. Shoeibi S, Mashreghi M., Biosynthesis of selenium nanoparticles using Enterococcus faecalis and evaluation of their antibacterial activities. J Trace Elem Med Biol. 2017, (39): 135-139

24. Jackman JA, Lee J, Cho NJ., Nanomedicine for infectious disease applications: innovation towards broadspectrum treatment of viral infections. Small. 2016, 12(9):1133-1139.

25. Hamdi Trabelsi, Inès Azzouz, Soumaya Ferchichi, Olfa Tebourbi, Mohsen Sakly, Hafedh Abdelmelek., Nano-toxicological evaluation of oxidative responses in rat nephrocytes induced by cadmium. International Journal of Nano-medicine. 2013, 8:3447-3453

26. Wenjuan Xun, Liguang Shi, Wenbin Yue, Chunxiang Zhang, Youshe Ren, Qiang Liu., Effect of high-dose nano-selenium and selenium-yeast on feed digestibility, rumen fermentation, and purine derivatives in sheep. Biol Trace Elem Res. 2012, 150(1-3):130-136.
27. Serra AB, Nakamura K, Matsui $T$, Harumoto H, Fujihara T., Inorganic selenium for sheep I. Selenium balance and selenium levels in the different ruminal fluid fractions. Asian J Anim Sci. 1994, 7:83-89.

28. Whanger PD, Weswing PH. Oldfield JE., Selenium, sulfur and nitrogen levels in ovine rumen microorganisms. Journal of Animal Science. 1978, 46:515-519.

29. Jannes BJ, Deagen JT, Beilstein MA, Whanger PD., Comparative metabolism of organic and inorganic selenium by sheep. J Agric Food Chem. 1989, 37:1358-1363.

30. Hidiroglou M, Lessard JR., The effect of selenium or vitamin E supplementation on volatile fatty acids content of rumen liquor in sheep fed a purified diet. Int. J. Vitam. Nutr. Res. 1976, 46:458-463.

31. Naziroglu M, Aksakal M, Cay M, Celik S., Effects of vitamin $\mathrm{E}$ and selenium on some rumen parameters in lambs. Acta Vet. Hung. 1997, 45:447-456.

32. Kheradmand E, Rafii F, Yazdi MH, Sepahi AA, Shahverdi AR, Oveisi MR., The antimicrobial effects of selenium nanoparticle-enriched probiotics and their fermented broth against Candida albicans. Daru. 2014, 22(1):48.

33. Faixová Z, Piešová E, Maková Z, Čobanová K, Faix Š., Effect of dietary supplementation with selenium-enriched yeast or sodium selenite on ruminal enzyme activities and blood chemistry in sheep. Acta Vet. Brno 2016, 85:185-194.

34. Mahan DC, Cline TR, Richert B., Effects of dietary levels of selenium-enriched yeast and sodium selenite as selenium sources fed to growing-finishing pigs on performance, tissue selenium, serum glutathione peroxidase activity, carcass characteristics, and loin quality. J Anim Sci. 1999, 77:2172-2179.

35. Spears JW., Trace mineral bioavailability in ruminants. J Nutr. 2003, 133:1506S1509S.

36. NRC. Nutrient Requirement for Beef Cattle 8th ed. National Academy Press, Washington, DC, USA, 2000. 
37. Giadinis ND, Loukopoulos P, Petridou, EJ, Panousis N, Konstantoudaki K, Filioussis G, Tsousis G, Brozos C, Koutsoumpas AT, Chaintoutis SC and et al., Abortions in three beef cattle herds attributed to selenium deficiency. Pak. Vet. J. 2016, 36:145-148.

38. McElwee K, Hoffmann R., Growth factors in early hair follicle morphogenesis. Eur. J. Dermatol. 2000, 10, 341-350.

39. Ristow M, Schmeisser S., Extending life span by increasing oxidative stress. Free Radic. Biol. Med. 2011, 51, 327-336,

40. Xiaoying $\mathrm{Wu}$, Jiguang Yao, Zisheng Yang, Wenbin Yue, Youshe Ren, Chunxiang Zhang, Xiaoni Liu, Huisheng Wang, Xingcai Zhao, Suying Yuan, Qian Wang, Liguang Shi, Lei Shi., Improved fetal hair follicle development by maternal supplement of selenium at nanosize (Nano-Se). Livestock Science 2011, 142(1):270-275.

41. Youcef Mehdi, Jean-Luc Hornick, Louis Istasse, Isabelle Dufrasne., Selenium in the Environment, Metabolism and Involvement in Body Functions. Molecules 2013, 18, 3292-3311

42. Petrie HT, Klassen LW, Klassen PS, O’Dell JR, Kay HD., Selenium and the immune response: 2. Enhancement of murine cytotoxic T-lymphocyte and natural killer cell cytotoxicity in vivo. J. Leukoc. Biol. 1989, 45:215-220.

43. Rasool Kachuee, Hossein Abdi-Benemar, Yaghoub Mansoori, Pedro SánchezAparicio, Jamal Seifdavati, Mona M. M. Y. Elghandour, Regulo Jiménez Guillén, Abdelfattah Z. M. Salem., Effects of Sodium Selenite, L-Selenomethionine, and Selenium Nanoparticles During Late Pregnancy on Selenium, Zinc, Copper, and Iron Concentrations in Khalkhali Goats and Their Kids. Biological Trace Element Research, 2019
44. Gholam Ali Kojouri, Samane Jahanabadi, Mojtaba Shakibaie, Ali Mohammad Ahadi, Ahmad Reza Shahverdi., Effect of selenium supplementation with sodium selenite and selenium nanoparticles on iron homeostasis and transferrin gene expression in sheep. Research in Veterinary Science. 2012, 93:275-278

45. Traulsen H, Steinbrenner H, Buchczyk DP, Klotz LO, Sies H., Selenoprotein P protects low-density lipoprotein against oxidation. Free Radic. Res. 2004, 38:123-128.

46. Sirous Sadeghian, Gholam Ali Kojouri, Abdonnaser Mohebbi., Nanoparticles of selenium as species with stronger physiological effects in sheep in comparison with sodium selenite. Biol Trace Elem Res. 2012, 146:302-8.

47. Muller AS, Pallauf J, Most E., Parameters of dietary selenium and vitamin $\mathrm{E}$ deficiency in growing rabbits. Journal of Trace Elements in Medicine and Biology. 2002, 16(1):47-55.

48. Sylvie Skalickova, Vedran Milosavljevic, Kristyna Cihalova, Pavel Horky, Lukas Richtera, Vojtech Adam., Selenium nanoparticles as a nutritional supplement. Nutrition 33 (2017) 83-90

49. NRC. Nutrient Requirements of Dairy Cattle, 7th Revised ed. National Academy Press, Washington, DC, USA, 2001.

50. NRC. Nutrient Requirements of Sheep, 6th ed. National Academy Press, Washington, DC, USA, 1985.

51. Papazafeiriou AZ, Lakis C, Stefanou S, Yiakoulaki M, Mpokos P, Papanikolaou K., Trace elements content of plant material growing on alkaline organic soils and its suitability for small ruminant extensive farming. Bulg. J. Agric. Sci. 2016, 22:733-739. 